TITLE:

\title{
Fast response of InSb Schottky detector
}

$\operatorname{AUTHOR}(\mathrm{S})$ :

Kanno, I; Hishiki, S; Kogetsu, Y; Nakamura, T; Katagiri, M

\section{CITATION:}

Kanno, I ... [et al]. Fast response of InSb Schottky detector. REVIEW OF SCIENTIFIC INSTRUMENTS 2007, 78(5): 056103.

ISSUE DATE:

2007-05

URL:

http://hdl.handle.net/2433/50140

\section{RIGHT:}

Copyright 2007 American Institute of Physics. This article may be downloaded for personal use only. Any other use requires prior permission of the author and the American Institute of Physics. 


\title{
Fast response of InSb Schottky detector
}

\author{
Ikuo Kanno, Shigeomi Hishiki, ${ }^{\text {a) }}$ and Yoshitaka Kogetsu \\ Graduate School of Engineering, Kyoto University, Sakyo, Kyoto 606-8501, Japan
}

Tatsuya Nakamura and Masaki Katagiri

Tokai Establishment, Japan Atomic Energy Agency, Tokai, Ibaraki, 319-0011, Japan

(Received 13 February 2007; accepted 16 April 2007; published online 11 May 2007)

An InSb Schottky detector, fabricated from an undoped InSb wafer with Hall mobility which is higher than those of previously employed InSb wafers, was used for alpha particle detection. The output pulse of this InSb detector showed a very fast rise time, which was comparable with the output pulses of scintillation detectors. (c) 2007 American Institute of Physics.

[DOI: 10.1063/1.2737768]

For the purpose of developing radiation detectors, especially photon detectors, with higher detection efficiency and better energy resolution than $\mathrm{Si}$ and Ge detectors, the compound semiconductor $\mathrm{InSb}$ is a promising candidate for use as a detector substrate. The atomic numbers (In:49, Sb:51) and density $\left(5.78 \mathrm{~g} \mathrm{~cm}^{-3}\right)$ of InSb predict nearly the same photon absorption efficiency with those of CdTe and CdZnTe, which is $400-1000$ times and 7-10 times greater than those of $\mathrm{Si}$ and $\mathrm{Ge}$, respectively. The band gap energy of $\mathrm{InSb}$ is $0.165 \mathrm{eV}$, which is the smallest among developed semiconductors. By assuming the same Fano factor as those of $\mathrm{Si}$ and Ge detectors, this band gap energy of InSb affords a twofold higher energy resolution than those of conventional semiconductor detectors. The high energy resolution predicted by the band gap energy puts the InSb detector at a certain disadvantage as it requires a cooling system for operation as a radiation detector. This is the main reason why no activity on the development of an InSb detector has been performed prior to our study, although McHarris pointed out the feasibility of $\mathrm{InSb}$ as radiation detector substrate. ${ }^{1}$

Another advantage of InSb is the high mobility of electrons and holes: 78000 and $750 \mathrm{~cm}^{2} \mathrm{~V}^{-1} \mathrm{~s}^{-1}$ at $77 \mathrm{~K}$ for electrons and holes, respectively, which is 50 and 5 times greater than those of $\mathrm{Si}$. A fast charge collection time and high counting rate could be expected when using $\mathrm{InSb}$ detectors.

So far, conventional $p n$ junction detectors were fabricated from $p$-type InSb substrates, ${ }^{2}$ and Schottky detectors were made from both $p$-type ${ }^{3}$ and undoped-type InSb substrates $^{4}$ using commercially available InSb wafers. Although these detectors are not at the point of discussing their energy resolution, alpha particles of ${ }^{241} \mathrm{Am}$ were detected at the operating temperature range from 2 to $115 \mathrm{~K}^{2}$ In one application of $\mathrm{InSb}$ detectors, neutrons were detected at $4.2 \mathrm{~K}$, ${ }^{5}$ using the nuclear reaction of $n+{ }^{3} \mathrm{He} \rightarrow p+t$. Moreover, energy peaks were observed in gamma ray measurements using a ${ }^{133} \mathrm{Ba}$ source. ${ }^{6}$ In the experiments of alpha particle detection described above, the rise times of the preamplifier output pulses were measured. The observed rise

\footnotetext{
${ }^{a)}$ Present address: Takasaki Establishment, Japan Atomic Energy Agency,
} Takasaki, Gunma 370-1292, Japan times were, however, slower than those of typical $\mathrm{Si}$ and $\mathrm{Ge}$ detectors.

In this article, we report on a Schottky detector made from a commercial undoped-type InSb wafer, which has a higher Hall mobility than in previously used $\mathrm{InSb}$ wafers. The signals obtained using this detector was very fast when compared with those of conventional semiconductor detectors.

The InSb wafer used was an undoped type, with a thickness of $0.5 \mathrm{~mm}$ and diameter of $2 \mathrm{in}$., manufactured by Wafer Technology, England (W.T.). The InSb wafer was cut into pieces with dimensions of nearly $12 \times 15 \mathrm{~mm}^{2}$. The electronic property measurements were carried out with changing temperature from 4.2 to $80 \mathrm{~K}$. The Hall mobility showed a higher value than those of previously employed InSb wafers, i.e., undoped $\mathrm{InSb}$ wafer manufactured by Sumitomo Electric Industries (S.E.I.) and Ge-doped $p$-type InSb wafer by W.T., as shown in Fig. 1. The present undoped InSb wafer showed slightly $n$ type in conductivity.

A Schottky detector was fabricated with the same method as described in Ref. 6, but $\mathrm{Au}-\mathrm{Pd}(40 \%)$ was employed for Schottky contact with the thickness of $12 \mathrm{~nm}$. The schematic drawing of the detector is shown in Fig. 1 of Ref. 6 . The resistivity of this detector was estimated from the current-voltage curve as $4.4 \mathrm{k} \Omega$ at the temperature of $4.2 \mathrm{~K}$. The detector was mounted on the cold stage of a cryostat (Infrared Co.), together with an alpha particle source, ${ }^{241} \mathrm{Am}$. Alpha particles were detected using the InSb detector with changing operating temperature. No bias voltage was applied due to the occurrence of large electrical noise: this was caused by the low resistivity of the InSb detector, as observed in previous experiments.

A typical output pulse obtained using a commercial charge-sensitive preamplifier (2003BT, Canberra, USA) is shown in Fig. 2. The very sharp line seen at $3 \mu$ s was taken as the contribution of the electrons. The following slow rise and fall are due to the contribution of the holes. The fast moving electrons were not properly integrated with this preamplifier: this preamplifier was previously developed for $\mathrm{Si}$ and $\mathrm{Ge}$ detectors with typical electron mobilities of 1500 and $3900 \mathrm{~cm}^{2} \mathrm{~V}^{-1} \mathrm{~s}^{-1}$, respectively; on the other hand, the electron mobility of this InSb was greater than $10^{4} \mathrm{~cm}^{2} \mathrm{~V}^{-1} \mathrm{~s}^{-1}$. With the same preamplifier, the energy of the alpha particles 


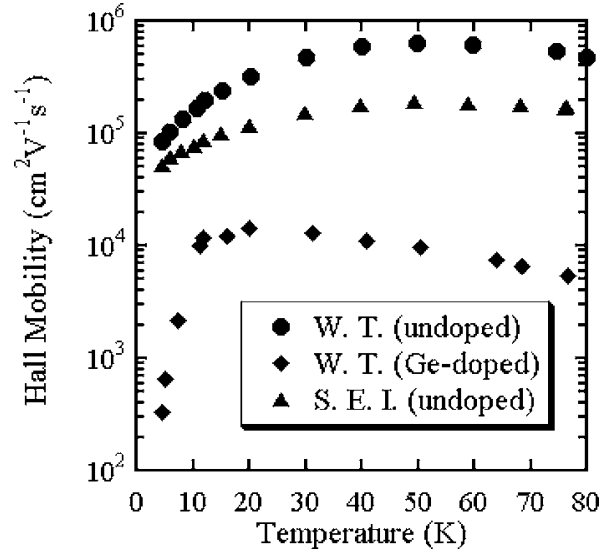

FIG. 1. Hall mobility of InSb wafers. Circles: undoped type, Wafer Technology (W.T.); diamonds: Ge doped $p$ type, W.T.; triangles: undoped type, Sumitomo Electric Industries (S.E.I.).

was measured using InSb detectors prepared from undoped (S.E.I.), and Ge-doped (W.T.) InSb wafers, with Hall mobilities of nearly $5 \times 10^{4}$ and $300 \mathrm{~cm}^{2} \mathrm{~V}^{-1} \mathrm{~s}^{-1}$ at $4.2 \mathrm{~K}$, respectively. This indicates that the electron mobility of the present $\mathrm{InSb}$ (W.T.) is much greater than that of the undoped $\mathrm{InSb}$ manufactured by S.E.I.

As a current-sensitive preamplifier, SA-430F5 (NF Corp., Japan) was employed. The obtained typical output pulse is shown in Fig. 3. The rapid rise time of nearly $3 \mathrm{~ns}$ is due to the contribution of electrons. The slower part following electron contribution is attributed to the holes. The total time from rise to decay was nearly $20 \mathrm{~ns}$.

This preamplifier covers the bandwidth from $400 \mathrm{~Hz}$ to $110 \mathrm{MHz}$. The preamplifier output pulses were observed using a digital storage oscilloscope with the bandwidth of $2 \mathrm{GHz}$. The rise time of observed pulses, $t_{\mathrm{obs}}$, is expressed by the following equation:

$$
t_{\mathrm{obs}}=\sqrt{t_{p}^{2}+t_{\mathrm{osc}}^{2}} .
$$

Here, $t_{p}$ is the rise time of the pulse, and $t_{\mathrm{osc}}$ is the rise time of the oscilloscope and is negligible in this case. The relationship between the rise time, $T$, and the bandwidth of the measuring device, $f_{c}$, is ${ }^{7}$

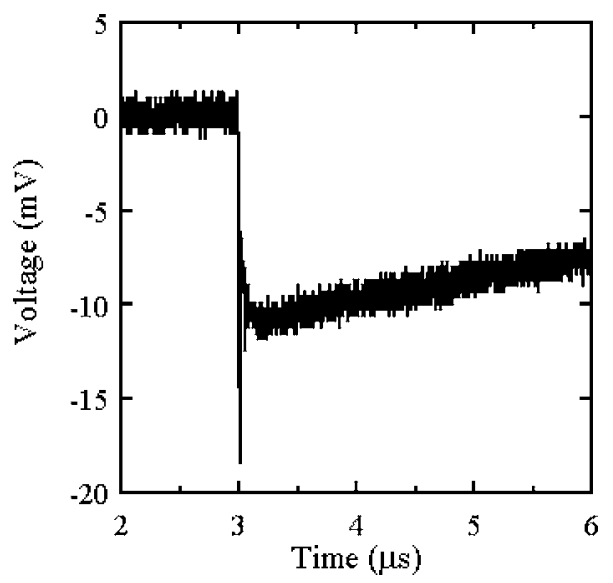

FIG. 2. Typical output pulse of alpha particles measured with the InSb Schottky detector at $4.2 \mathrm{~K}$, using a charge-sensitive preamplifier (2003BT, Canberra).

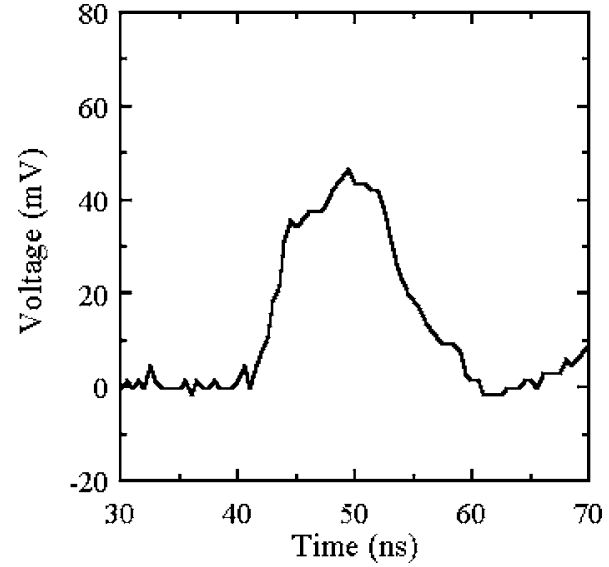

FIG. 3. Typical output pulse of alpha particles measured with the InSb Schottky detector at $4.2 \mathrm{~K}$, using a current-sensitive preamplifier (SA430F5, NF Corp.)

$$
f_{c}=0.35 / T \text {. }
$$

In the case of measuring pulses with rise times of $3 \mathrm{~ns}$, a bandwidth of $117 \mathrm{MHz}$ is necessary. Therefore, the observed rise time of the pulse was mainly determined by the bandwidth of the preamplifier. We can conclude that the real rise time of the charge movement in the InSb detector was faster than 3 ns.

The observed rise time of this InSb detector, as well as other previously fabricated InSb detectors, ${ }^{8}$ is shown as a function of operating temperature in Fig. 4. The observed rise time of 3 ns is very fast. Even in the case of recognizing the rise-decay time of $20 \mathrm{~ns}$ as the rise time in the chargesensitive preamplifier, it is very short compared to those of Ge and Si drift detectors, 130 and $80 \mathrm{~ns},{ }^{9}$ respectively, and is comparable with the rise time of fast scintillators. This short rise time of the InSb detector will be useful for the measurements with high counting rate in, for example, $\mathrm{x}$-ray fluorescence analysis.

This work was supported by Grant-in-Aid for Scientific Research funding from the Japan Society for Promotion of Science, and the Iketani Science and Technology Foundation.

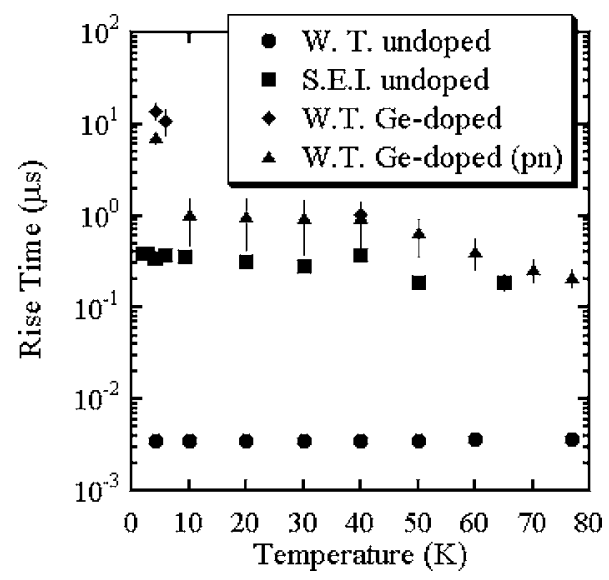

FIG. 4. Rise time of InSb detectors during alpha particle detection as a function of operating temperature. Circles: undoped (W.T.) Schottky detector; squares: undoped (S.E.I.) Schottky detector; diamonds: Ge doped $p$-type (W.T.) Schottky detector; and triangles: Ge doped $p$-type (W.T.) $p n$ junction detector. 
${ }^{1}$ Wm. C. McHarris, Nucl. Instrum. Methods Phys. Res. A 242, 373 (1986).

${ }^{2}$ I. Kanno, F. Yoshihara, R. Nouchi, O. Sugiura, Y. Murase, T. Nakamura, and M. Katagiri, Rev. Sci. Instrum. 74, 3968 (2003).

${ }^{3}$ I. Kanno, F. Yoshihara, R. Nouchi, O. Sugiura, T. Nakamura, and M. Katagiri, Rev. Sci. Instrum. 73, 2533 (2002).

${ }^{4}$ I. Kanno, S. Hishiki, O. Sugiura, R. Xiang, T. Nakamura, and M. Katagiri, Nucl. Instrum. Methods Phys. Res. A 568, 416 (2006).

${ }^{5}$ T. Nakamura, M. Katagiri, Y. Aratono, I. Kanno, S. Hishiki, O. Sugiura, and Y. Murase, Rev. Sci. Instrum. 75, 340 (2004).
${ }^{6}$ S. Hishiki, Y. Kogetsu, I. Kanno, T. Nakamura, and M. Katagiri, Nucl. Instrum. Methods Phys. Res. A 559, 558 (2006).

${ }^{7}$ D. L. Schilling and C. Belove, Electronic Circuits: Discrete and Integrated (McGraw-Hill, New York, 1968).

${ }^{8}$ S. Hishiki, I. Kanno, O. Sugiura, R. Xiang, T. Nakamura, and M. Katagiri, IEEE Trans. Nucl. Sci. 52, 3172 (2005).

${ }^{9}$ M. A. El-Wahab and A. El-Arabi, IEEE Trans. Nucl. Sci. 40, 147 (1993); C. Fiorini, A. Gola, A. Longoni, F. Perotti, and L. Strueder, ibid. 51, 1091 (2004). 